\title{
Serial-pattern structure and temporal-order recognition
}

\author{
MARILYN BOLTZ, ELIZABETH MARSHBURN, MARI RIESS JONES, \\ and WALTER W. JOHNSON \\ The Ohio State University, Columbus, Ohio
}

\begin{abstract}
Two studies investigated the respective roles of pattern contour (number of contour changes), rule invariance, contour shape, and rate upon the recognition of 8-tone auditory sequences. Tonal patterns contained $0,1,2$, or 3 contour changes, which were introduced in conjunction with a randomly selected musical interval (rule-variant patterns) or with predictable (rule-governed) musical transformations (rule-invariant patterns). Patterns were either symmetrical or asymmetrical in shape. Listeners discriminated transposed standards from distractor patterns that contained an order reversal. In Experiment 1, where patterns occurred at a slow rate, performance decreased as number of contour changes increased. No effects of rule invariance or contour shape were found. In Experiment 2, where patterns occurred at a rate twice that of Experiment 1, more contour changes again had a detrimental effect. In addition, rule-invariant patterns were easier than rule-variant patterns. Results suggest that contour contributes to temporal order confusion in a systematic way.
\end{abstract}

Over the last several years, a small, but substantial, body of research has suggested that directional changes in frequency have important effects upon auditory pattern recognition. Using a STM task, Dowling and Fujitani (1971) initiated this research with randomly generated tone patterns. They found that listeners confused sequences that had the same contour (i.e., patterns of "ups" and "downs" in pitch) even though these sequences differed with respect to interval properties (i.e., pitch distance relations). More recent research by Dowling (e.g., 1972, 1978a, 1978b; Dowling \& Bartlett, 1981) verified that contour has an important influence on melody recognition, particularly at brief retention intervals. And, in a separate series of studies, Massaro and his colleagues (Idson \& Massaro, 1978; Kallman \& Massaro, 1979; Massaro, Kallman, \& Kelly, 1980) have demonstrated that contour can affect tune recognition. In extending work of Deutsch (1972), they asked listeners to recognize distortions of familiar tunes whose notes had been randomly assigned to different octaves. Results showed that recognition was impaired when the random-note assignments violated contour. Other studies using incidental learning tasks have reported that contour complexity contributes to recognition difficulty (e.g., Cuddy, Cohen, \& Mewhort, 1981).

One curious aspect of much of this research has been the experimental approach to the manipulation of contour.

This research was supported by Grant BNS-820481 I from the National Science Foundation. The authors thank Barbara Fisher and Erin Cassidy for assistance in data collection. Requests for reprints should be sent to Mari Riess Jones, Department of Psychology, The Ohio State University, 404C West 17th Avenue, Columbus, OH 43210.
Studies have tended to use melodic sequences whose contour manipulations are confounded with pitch-interval relationships. Indeed, studies that independently vary both contour and pitch-interval relationships are infrequent. An exception is a study by Cuddy et al. (1981), wherein musical conventions were used to construct patterns with specific pitch-interval and contour properties. Even so, the primary purpose of that study was not a parametric evaluation of contour.

In contrast, the present studies systematically manipulated both pitch intervals and contour. Special constraints were placed upon the construction of tonal sequences such that effects due to contour shape, number of contour changes, and pitch intervals could be independently assessed. The aim of these studies was to examine effects of contour and melodic rule structure upon perceived event order in pattern recognition.

Traditionally, loss of perceived temporal order has been associated almost exclusively with the temporal variable of pattern rate. It has been common to assume that order confusions are primarily a function of the time interval or stimulus-onset-asynchrony (SOA) interval between adjacent events. Specifically, it has been suggested that order confusions arise when some limiting rate is approached or exceeded (e.g., Fay, 1966; Hirsh \& Sherrick, 1961; Stroud, 1955; Warren, 1974, 1976). There is reason to believe, however, that nontemporal aspects of pattern structure, including both contour and interval-pitch structure, also contribute to temporal order confusions (cf. Boltz \& Jones, 1985; Divenyi \& Hirsh, 1974; Hirsh, 1974).

Contour may affect order retention because changes in pitch direction effectively segment the structure of serial patterns into temporal segments. In auditory sequences 
composed of different frequencies, these segments form unidirectional pitch trajectories that move invariantly "up" (higher in pitch) or "down" (lower in pitch). Thus, consider a sequence of tones, such as:

$$
\mathrm{C}_{4}{ }^{+} \mathrm{D}_{4}{ }^{+} \mathrm{F}_{4}{ }^{+} \mathrm{G}_{4}^{-} \mathrm{E}_{4}^{-} \mathrm{D}_{4}^{-} \mathrm{B}_{3}^{-} \mathrm{A}_{3}
$$

(in musical notation, where subscription identifies octave: $\mathrm{C}_{4}=$ middle $\mathrm{C}$ on the piano $=262 \mathrm{~Hz}$ ). This sequence is segmented by pitch contour into two subgroups, an initial one moving up, +++ (from $\mathrm{C}_{4}$ to $\mathrm{G}_{4}$ ), and a second one moving down, - - - (from $G_{4}$ to $\left.A_{3}\right)$. If a listener is sensitive to this up-down shape, then the ordering of pitches will necessarily be correlated with these groupings. Furthermore, notice that the temporal arrangement of tones within these groups cannot be changed without also tampering with overall contour. For example, if the events $\mathrm{D}_{4}$ and $\mathrm{F}_{4}$ are reversed in time, the contour for the resulting sequence, $C_{4} F_{4} D_{4} G_{4} E_{4} D_{4} B_{3} A_{3}$, becomes +-+---- . Thus, contour, pitch, and temporal-order properties of a sequence are inextricably bound together. If a person attends to contour and related pitch trajectories over time, then the person is also attending to temporal order information.

It is also reasonable to suppose that the pitch and contour relationships of some patterns may be more predictable than others because of an underlying rule structure. For example, if a contour change always involves a given degree of pitch change relative to a preceding tone, this may attenuate any potential detriment resulting from the contour break. Or, if the set of tones within a contourdefined segment are lawfully related by a common pitch rule, this might similarly improve performance. This is because the serial order of tones within a given contour segment is essentially dictated by successive pitch transformations that relate both adjacent and remote tones and that stem from a common tonal referent (Jones, 1974, 1976; Restle, 1970). For example, consider the following sęquence:

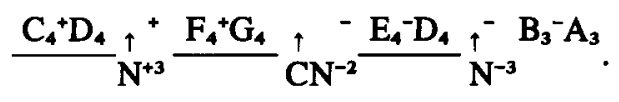

The opening tonal argument, $\mathrm{C}_{4} \mathrm{D}_{4}$, involves a lower order rule, $\mathrm{N}^{+1}$, of one scale-step shift (e.g., from $\mathrm{C}$ to $\mathrm{D}$ in the 4th octave, where 4 subscripts octave). This lower order rule remains invariant in the pattern across 2-tone groups but is broken by higher order rules relating groups (e.g., between $\mathrm{D}_{4}$ and $\mathrm{F}_{4}$ ). This initial phrase here is transformed $\left(\_\uparrow\right)$ into $F_{4} G_{4}$ by the higher order $N^{+3}$ rule, thereby adding three scale steps to each note. The remaining notes are generated by other rules (e.g., $\left.\mathrm{CN}^{-2}, \mathrm{~N}^{-3}\right)^{1}$ applied to immediately preceding tonal groups. Some of these rules involve complementation on the scale (i.e., $\mathrm{CN}^{-2}$ ) and can produce contour changes (see Figure 1 for rule definitions). Because of its higher order rule structure, this sequence exhibits a high degree of predictability in both contour and pitch-interval relationships. If peo-

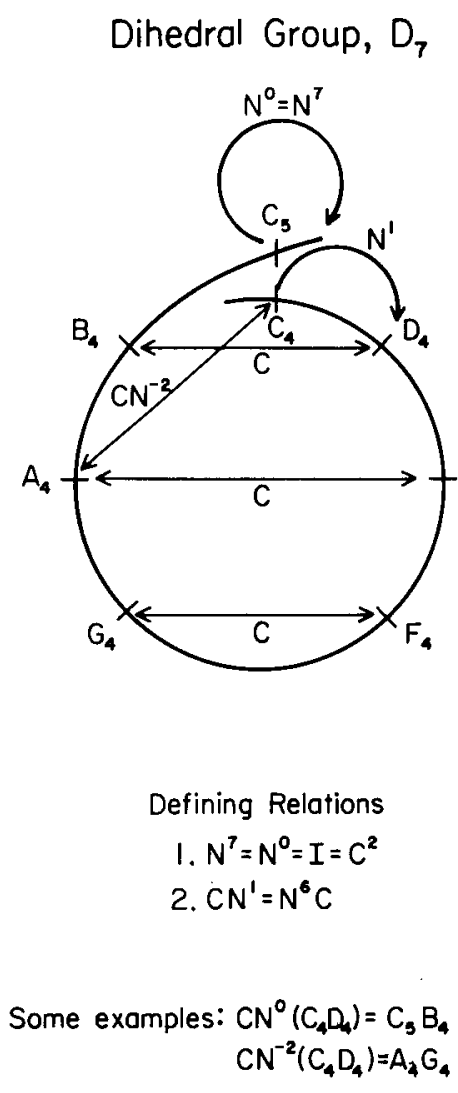

Figure 1. Dihedral group, $D_{\text {, }}$, used to generate melodies. Rules are $\mathrm{N}$ and $\mathrm{CN}$, which involves complementation (C) followed by $\mathrm{N}$ on the circular alphabet.

ple are sensitive to this predictability, then order reversals may be more distinctive in these rule-invariant patterns.

Assuming that rule-invariant patterns do promote superior order retention, we now need to consider whether this facilitation is due to regularities in contour, pitchinterval relationships, or both. Can the presence of contour predictability by itself yield superior order retention? To answer this question, we need to compare performance on patterns that lack pitch-interval predictability but which contain the same contour as rule-invariant patterns. For example, consider the following melody:

$$
\mathrm{C}_{4}{ }^{+} \mathrm{E}_{4}{ }^{+} \mathrm{F}_{4}{ }^{+} \mathrm{B}_{4}-\mathrm{G}_{4}{ }^{-} \mathrm{E}_{4}-\mathrm{C}_{4}{ }^{-} \mathrm{A}_{3} \text {. }
$$

This pattern lacks a higher order (interval) rule structure and so its pitch-interval relationships are less predictable (i.e., more variable). The contour of this pattern, however, is identical to the rule-invariant pattern illustrated earlier. Since there is only one contour change, and overall contour shape is quite symmetrical (i.e., $\Lambda$ ), order reversals within this sort of pattern may also be quite distinctive-even though pitch-interval predictability is lacking. 
In short, we are suggesting that if rule invariance does facilitate order retention, this may be simply because the predictability in pitch intervals is correlated with predictability of contour information. This emphasis on contour contrasts with a more traditional approach to serial-order retention. Some theorists have suggested that more complex memory codes are associated with rule-variant patterns and that code complexity may impair order retention (e.g., Restle, 1970; Restle \& Brown, 1970). Rule invariance should therefore facilitate performance, because order information is more simply coded and more easily accessed. It is important to note, however, that ruleinvariant patterns are predicted to produce superior performance, regardless of pattern contour. This is because Restle's theory of serial coding ignores covarying effects of pitch interval and contour information. Instead, order retention is attributed entirely to rule regularity.

The present experiments were designed primarily to investigate the influence of a pattern's contour and its pitchrule structure upon people's ability to identify the temporal order of its tones. In particular, we wished to investigate whether pitch-rule invariance was the sole determinant of order retention (as Restle claims), or whether performance was qualified by contour shape and number of contour changes. The variable of rate was also studied by contrasting patterns of a slow rate (Experiment 1) with patterns of a faster rate (Experiment 2).

The studies to be reported evolved from a series of preliminary experiments in which both the number of contour changes and the type of contour change (predictable and unpredictable pitch intervals) were studied in tonal patterns of lengths ranging from 6 to 10 tones. These earlier experiments suggested that contour complexity, as measured by number of contour changes, has detrimental effects on pattern recognition performance, but that these effects can occasionally be attenuated by introducing structural redundancy in the form of predictable pitch intervals. The two studies reported here rigorously explored these preliminary findings, and considered their possible theoretical implications.

Auditory patterns used in the present studies were generated from arrangements of 8 tones drawn from a familiar musical scale, the $\mathrm{C}$ major scale (see Footnote 2 for musical definitions). Sequences always contained 0 , 1,2 , or 3 contour changes, but in half, specific serial rules were applied to create lawful sequences of invariant melodic relationships (rule invariant). The remaining sequences possessed contours identical to rule-based counterparts but lacked lawful rule structure (rule variant). Thus, the rule-invariant melodies incorporated substantial predictability with respect to pitch intervals. If pitch contour contributes to tonal grouping and hence to order retention, then, as more contour changes complicate a pattern, order retention should decline correspondingly for patterns with and without rule regularity. In addition, any qualifying effects of rule variability and rate upon this effect of contour were of interest. These effects were evaluated here using a two-alternative forced-choice task
(2AFC), in which the decoy alternative pattern always contained an order reversal.

Finally, another variable was included in the present studies to assess the impact of pattern shape and recursive combinations of interval rules upon order retention. To this end, half the patterns (in each experiment) possessed an overall symmetrical contour with respect to the placement of contour changes in each pattern. For instance, in patterns with a single contour change, symmetrical instances exhibit two 4-tone groups, as in

$$
0^{\circ} \circ^{\circ}
$$

whereas their asymmetrical counterparts have a skewed shape as in

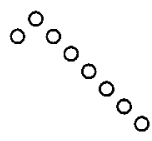

This variable of contour shape was, by necessity, differentially developed for rule-invariant and rule-variant patterns. In rule-invariant patterns, symmetrical contours resulted from a symmetrical arrangement of higher order transformational rules that served to relate both adjacent and remote tones. The pattern-generation procedure relied upon well-known rule-recursive techniques popularized by Restle, in which a tonal argument (e.g., CD) is transformed and concatenated to form larger sequences. Thus, in

$$
\frac{\mathrm{C}_{4} \mathrm{D}_{4}}{\mathrm{~N}^{+3}} \stackrel{\mathrm{F}_{4} \mathrm{G}_{4}}{\uparrow} \stackrel{\mathrm{CN}}{-2}^{\mathrm{E}_{4} \mathrm{D}_{4}} \underset{\mathrm{N}^{-3}}{\uparrow} \mathrm{B}_{3} \mathrm{~A}_{3},
$$

where $\mathrm{N}^{ \pm 3}$ flanks $\mathrm{CN}^{2}$, this pattern can also be rewritten recursively as $\mathrm{CN}^{-5}\left(\mathrm{~N}^{+3}(\mathrm{CD})\right.$ ), where $\mathrm{N}^{+3}(\mathrm{CD}) \rightarrow \mathrm{C}_{4}$ $\mathrm{D}_{4} \mathrm{~F}_{4} \mathrm{G}_{4}$ and $\mathrm{CN}^{-5}\left(\mathrm{C}_{4} \mathrm{D}_{4} \mathrm{~F}_{4} \mathrm{G}_{4}\right) \rightarrow \mathrm{C}_{4} \mathrm{D}_{4} \mathrm{~F}_{4} \mathrm{G}_{4} \mathrm{E}_{4}$ $\mathrm{D}_{4} \mathrm{~B}_{3} \mathrm{~A}_{3}$. Rule-recursive patterns have been also termed hierarchical patterns because the rules typically map into abstract hierarchical trees (Jones, 1981b; Restle, 1970). Hierarchical patterns, because of their rule-recursive property, also result in economical codes. Other ruleinvariant patterns lack rule recursion (i.e., their higher order rules are independent of each other) and should therefore produce more complex codes. These were termed asymmetric rule-invariant patterns because their asymmetrical arrangements of higher order rules yielded asymmetrical contour shapes.

In the rule-variant patterns, contour symmetry was manipulated by constructing patterns with globally symmetric pattern shapes and others with asymmetric shapes. These sequences lacked the pitch predictability of their rule-invariant counterparts, but they nevertheless contained contours that were perfectly matched to respective symmetric or asymmetric rule-invariant patterns. 
With respect to rule-invariant patterns, prevailing coding theories (e.g., Greeno \& Simon, 1974; Restle, 1970) suggest that serial ordering may be more concisely specified in sequences that lend themselves to hierarchical rewriting. Because codes corresponding to hierarchical patterns are less complex, Restle's theory predicts that performance should be better on rule-invariant patterns than on rule-variant ones and that it should be best of all for the hierarchical rule-invariant patterns.

On the other hand, others have reported pronounced performance differences among rule-structured melodies as a function of contour and have thus questioned the generality of rule recursion (Boltz \& Jones, 1985; Jones, Maser, \& Kidd, 1978). It is possible that beneficial effects of rule-recursive patterns (if present) do not stem from the specific arrangement of pitch intervals within the hierarchical rule-invariant sequences. They may derive merely from a general contour symmetry that is correlated with rule recursion. That is, perhaps order reversals are more apparent in any pattern with a symmetrical contour (which hierarchical patterns always exhibit). If this is so, it would pose problems for theories, such as Restle's, that place a premium on the particular interval distances in pitch. Beneficial effects due to pattern shape but not to underlying interval rules would mean that all symmetrical patterns, regardless of their rule structure, would lead to better performance than asymmetrical counterparts. In this case, then, we would expect a general superiority for symmetrical patterns with no interaction with rule structure.

Figure 2 presents examples of pattern instances from the 16 conditions appearing in Experiment 1 (slow rate) and Experiment 2 (fast rate). These conditions are defined by combinations of contour change $(0,1,2,3)$, rule (invariant, variant), and shape (symmetry, asymmetry). In both studies, listeners with modest musical experience judged which of two comparison sequences was identical in temporal-order arrangement to a standard pattern.

\section{EXPERIMENT 1}

\section{Method}

Design and Subjects. The design was a $4 \times 2 \times 2 \times 2$ mixed factorial involving four levels of contour change $(0,1,2,3)$, two levels of rule structure (invariant, variant), two levels of contour shape (symmetric, asymmetric), and two levels of counterbalance order. Counterbalance order served as the only between-subjects variable.

The subjects were 24 Ohio State University students, who volunteered to participate in a psychology experiment for course credit or for payment ( $\$ 2$ per hour). Each had played a musical instrument for at least 2 of the previous 5 years. Twelve subjects were assigned randomly to each of two counterbalance orders and tested in groups of one to four. (Two subjects failed to perform above chance levels and were discarded for statistical analysis.)

Procedure. Recorded instructions informed subjects of pattern presentation details and the requirements of a 2 AFC task. The subjects judged which of two comparison patterns was identical to a preceding standard pattern. Although the two comparisons were always transposed 1 octave higher than the standard, the subjects were instructed to ignore absolute-pitch differences and to concentrate upon the equivalency of melodic arrangement.

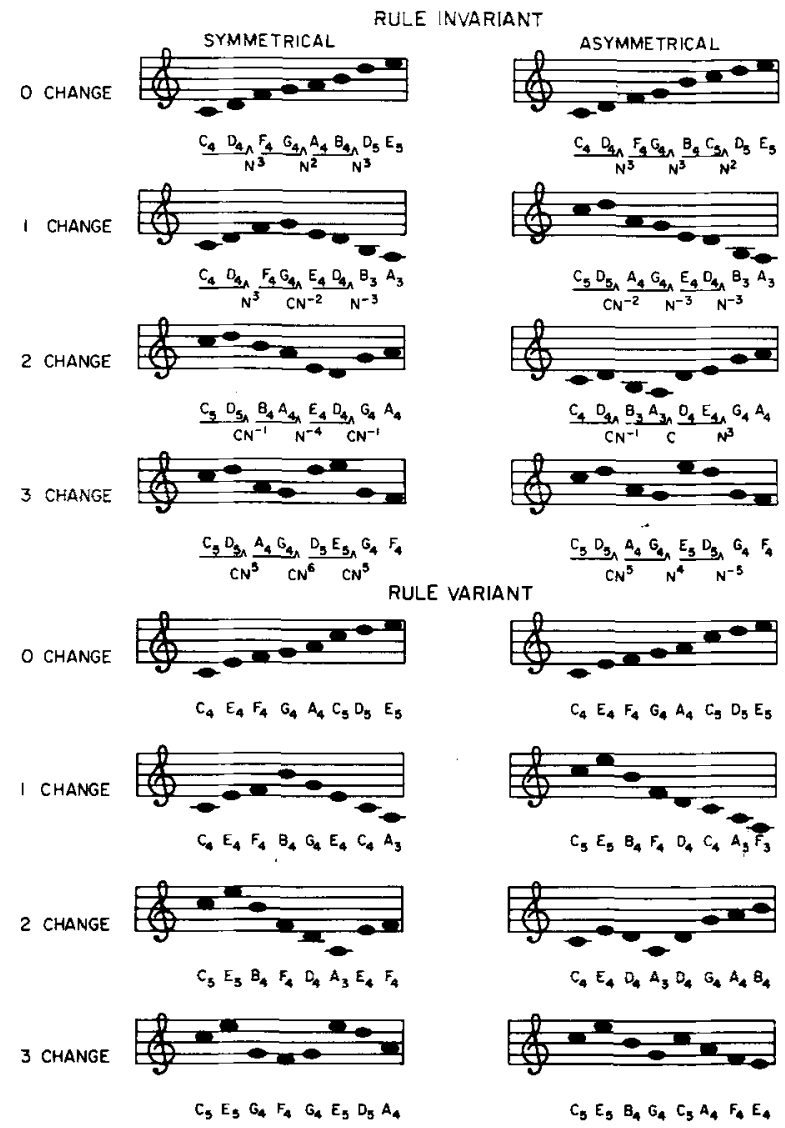

Figure 2. Examples of melodies used in Experiments 1 and 2. Ruleinvariant patterns, shown at four levels of contour change, were generated from higher order $\mathrm{CN}^{\mathrm{j}}$ rules to result in a symmetrical or asymmetrical pattern shape. Rule-variant patterns lacked a higher order rule structure, but preserved an identical contour to a ruleinvariant counterpart (see Footnote 1).

The subjects made judgments on a total of 128 successive trials (two blocks of 32 trials on each of 2 successive days). Each trial presented three 8-tone patterns (one standard and two comparisons). Each trial was signaled by a 1-sec warning tone $(5000 \mathrm{~Hz})$, which preceded the standard pattern by 2 sec. Subsequent patterns of the triad followed one another by $3 \mathrm{sec}$, with a $10-\mathrm{sec}$ response interval following the final comparison pattern. During the response interval, the subjects recorded their equivalence judgments, in writing, by checking a box that corresponded to either the first or second pattern.

At the beginning of each of two daily sessions, the subjects received five practice trials involving an assortment of different pattern types and contour changes. Altogether, each day's session lasted approximately $45 \mathrm{~min}$, with the subjects receiving a brief rest break (ca. $2 \mathrm{~min}$ ) between the first and second trial block.

Apparatus. All patterns were constructed of sine-wave tones generated by a Wavetek Model 159 waveform generator that was controlled by a Cromemco Z-2 microcomputer. In generation, 10msec rise and fall times were imposed on all tones by a custombuilt envelope generator.

Sequences of tones, programmed by the microcomputer, were recorded on a Nakamichi LX-3 cassette recorder. Sets of prerecorded sequences were presented through AKG- 90 headphones at a comfortable listening level. Tones themselves were subjectively equated for loudness in the range of the pitch intervals used. 
Stimulus materials. A total of 1928 -tone patterns were constructed and consisted of 64 standard and 128 comparison patterns. In all patterns, tonal durations were $300 \mathrm{msec}$ with a 50 - $\mathrm{msec}$ intertone interval, yielding uniform SOAs of $350 \mathrm{msec}$.

Standard patterms differed in rule type (invariant, variant), number of contour changes $(0,1,2,3)$, and contour shape (symmetric, asymmetric), yielding 16 standard pattern types. Four instances of each pattern type were constructed.

Rule-invariant patterns were generated for each of the four levels of contour change by applying a series of transformational rules to initial two-tone arguments $\left(\mathrm{C}_{4} \mathrm{D}_{4}, \mathrm{C}_{4} \mathrm{E}_{4}, \mathrm{C}_{5} \mathrm{~B}_{4}\right.$, and $\left.\mathrm{C}_{5} \mathrm{~A}_{4}\right)$. Rules were drawn from a well-known symmetry group, the dihedral group $\mathrm{D}_{7}$. As Figure 1 illustrates, generative rules of this group consist of next rules $\left(\mathrm{N}^{j}\right)$ and next rules compounded by complementation $\left(\mathrm{CN}^{j}\right)$. Their application is described in detail elsewhere (Boltz \& Jones, 1985). Two types of rule-invariant patterns differed in terms of pattern shape.

Sixteen rule-invariant patterns each displayed a symmetrical contour shape rendered by a hierarchical (rule recursive) arrangement of generative rules. This rule arrangement afforded recursive rewriting such that the second half of each pattern could be generated by a single rule transformation. ${ }^{1}$ In Figure 2, for example, the symmetrical rule arrangement of $\mathrm{N}^{+3} \circ \mathrm{N}^{+2} \circ \mathrm{N}^{+3}$ (where " $\mathrm{o}$ " means "followed by") that describes the 0 change condition can be recursively rewritten as $\mathrm{N}^{+5}\left(\mathrm{~N}^{+3}\left(\mathrm{C}_{4} \mathrm{D}_{4}\right)\right.$ ).

From the 16 symmetric rule-invariant patterns, 16 asymmetric counterparts were derived. Asymmetric sequences were produced by using, as much as possible, the same notes and rules as a corresponding symmetric sequence. Rules were rearranged, however, such that these patterns could not be recursively rewritten; in addition, they yielded a nonsymmetrical contour shape. Examples of asymmetric rule-invariant patterns, at each of the four contourchange conditions, are shown in Figure 2.

Lastly, for all symmetric and asymmetric rule-invariant patterns, a rule-variant counterpart was created. These patterns could not be described by a higher order generative rule structure, but did contain an identical contour to a rule-invariant counterpart.

In the construction of standard (and comparison) patterns, three constraints were imposed. First, all patterns were generated from notes of the $\mathrm{C}$ major scale, where $\mathrm{C}_{4}=262 \mathrm{~Hz}$. Second, no patterns contained trill-like relationships (i.e., G-F-G-F). Third, all patterns were equated, as closely as possible, on the following dimensions: (1) number of diffferent notes used, (2) range of pitcht intervals, and (3) the number of new contour changes introduced by the order reversals in comparison patterns (see below).

Comparison patterns in each condition were constructed for every standard pattern. All comparison patterns were transposed up 1 octave from the standard. Three kinds of comparison sequences were generated for each standard, one "same" comparison (i.e., the correct choice) and two "differents," although on any given trial only one of the "different" comparisons was presented. The two "different" comparisons differed from one another with respect to the serial location of a single order reversal of adjacent pattern tones. In half the "different" patterns, the tones reversed were those at serial positions 2 and 3 , and in the remaining half, were those at 5 and 6 . It should be mentioned that inevitably the introduction of order reversals into the "different" patterns changes their contour (shifts, adds, or removes a contour break). Since there were 64 standard melodies and since each standard appeared once with each of the two "different" sequences, this constituted a total of 128 trials.

On each trial, the subjects heard three patterns and half the time the correct alternative assumed the first position immediately following the standard. Trials were presented in random order, with the exception that the following pattern types were not allowed to occur consecutively: (1) patterns of a given rule type (rule invariant, rule variant) or contour shape (symmetric, asymmetric), (2) patterns with a given number of contour changes. (3) patterns with the same starting two notes, and (4) patterns with the same reversal location in the "different" pattern.

\section{Results and Discussion}

An analysis of variance was performed on the mean proportion of correct responses (PC), where the variables of interest were rule structure, number of contour changes, contour shape, and counterbalance order. Figure $3 \mathrm{a}$ shows the mean proportion correct pattern recognition as a function of rule structure (rule invariant, rule variant) and number of contour changes $(0,1$, $2,3)$. Results are collapsed over counterbalance order and contour shape, since both variables were nonsignificant.

The most important finding was that, as predicted, contour complexity had large and significant effects on judgments about these sequences $[\mathrm{F}(3,69)=16.90, \mathrm{MSe}=$ $.011, \mathrm{p}<.001]$. As number of contour changes increased, PC decreased. This was true for both ruleinvariant and rule-variant patterns. In fact, the main effect of the rule-structure variable contributed negligible variability $[F(1,23)<1.00]$, and this variable did not interact significantly with contour $[F(3,69)<1.00$ for the interaction].

The impact of contour upon order retention performance is clearly much greater in these data than the effects of rule structure. In fact, it is interesting that in some cases melodies with greater rule variability are actually easier than rule-invariant ones. These large effects of pattern contour, particularly in the absence of significant differences due to rule structure, are difficult for coding theories such as Restle's, in which order confusions depend simply upon rule structure and not upon contour.

The fact that patterns with pitch-interval unpredictability did not prove to be more difficult than those with more rule predictability appears to stem in part from ceiling effects. Especially with patterns having relatively few contour changes $(0,1)$, performance was extremely good with both the rule-invariant and rule-variant melodies. For these reasons, it may be more interesting to discover if rule-invariant patterns containing a greater number of contour changes $(2,3)$ were easier than their variant counterparts. In fact, however, performance on these patterns was also comparable. These results are not entirely consistent with the expectation that rule predictability attenuates order confusions.

There are also questions of pattern shape to consider. Do hierarchical rule patterns fare better than the asymmetric rule-invariant counterparts, as we might anticipate if rule recursion assists in establishing order over time? The answer is "no." Overall symmetrical patterns were not superior to asymmetrical ones $[F(1,23)<1.00]$, and this was true for both rule-invariant and rule-variant sequences $[F(1,23)<1.00$ for the interaction]. These findings at first glance seem to invalidate both the serial coding hypothesis that rule recursion cements serial order and the general contour symmetry argument that regularity of contour breaks enhances order recognition. There is, 

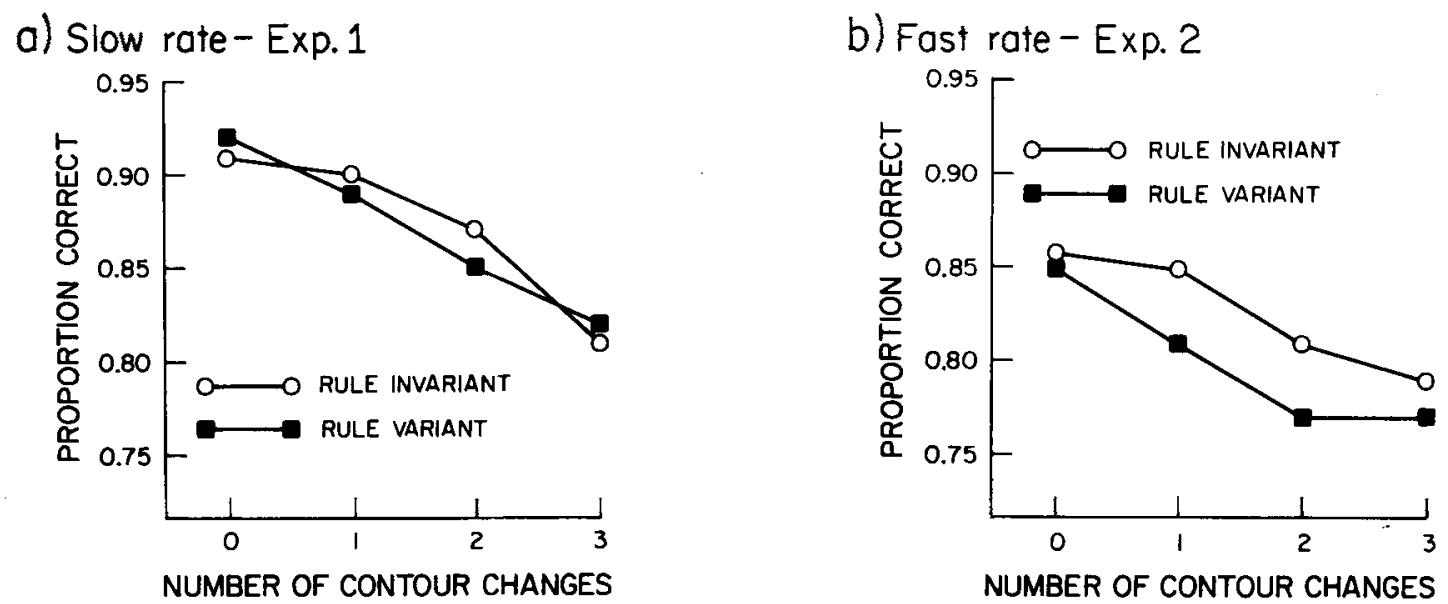

Figure 3. (a) Mean proportion of correct recognition in Experiment 1 (slow rate) as a function of rules and number of contour changes. (b) Mean proportion of correct recognition in Experiment 2 (fast rate) as a function of rules and number of contour changes.

however, modest and qualified support in these data for the rule-recursion proposal. It is found in a significant three-way interaction of pattern shape with rule level and contour $[\mathrm{F}(3,69)=3.85, \mathrm{MSe}=.009, \mathrm{p}<.025]$. The data are shown in Table 1.

This interaction stems from the fact that in rule-invariant patterns, asymmetrical pattern shape had a distinctly detrimental effect on patterns with three contour changes [Bonferroni $F(2,69)=2.84, p<.05]$. This effect of symmetry was not evident in the other rule-invariant contour conditions, nor was such a trend apparent in rulevariant patterns. Indeed, in the latter, the largest difference as a function of contour regularity emerged for the one contour change condition and favored patterns with asymmetrical contours $[\mathrm{F}(2,69)=2.52, \mathrm{p}<.05]$. Thus, it appears that the degree to which rule recursion helps performance depends significantly upon contour complexity. This, however, is limited support for the rulerecursion hypothesis, because the effect is qualified by number of contour changes and does not hold for all contour levels. This finding, then, is difficult for an approach such as Restle's, which does not incorporate a role for contour. It is, however, in agreement with other data (Boltz \& Jones, 1985) using a serial reproduction task.

In sum, temporal-order confusions in music-like patterns are unaffected by global contour shape, but do significantly increase with a greater number of contour changes. This happens both with sequences generated from an invariant (and hence predictable) series of tonal transformations and with sequences having substantially less predictability. The finding that contour complexity overwhelms effects of rule predictability poses problems for some coding models.

\section{EXPERIMENT 2}

Order confusions have been classically associated with the amount of time provided to resolve serial item succession. A common finding is that order confusions in- crease as time intervals (e.g., SOAs) decrease, and this has led to various hypotheses that emphasize the primary function of time thresholds (e.g., Stroud, 1955; Thomas, Hill, Carroll, \& Garcia, 1970; van Noorden, 1975; Warren, 1974, 1976). Presumably there exists a critical time interval (e.g., Stroud, 1955) or a required amount of processing time (Massaro, 1972; Warren, 1974) necessary for the identification and ordering of successive events (i.e., tones, phonemes, etc.). A problem has been the lack of agreement upon the size of a critical time interval for temporal order. Estimates range from values of 15 or $20 \mathrm{msec}$ for sequences of length 2 to $250 \mathrm{msec}$ (SOA) for longer sequences (Hirsh, 1959; Massaro, 1972).

Jones (1976) has suggested that one reason for this ambiguity is that an absolute temporal threshold does not exist. By positing a critical time interval, it is implied that time is solely responsible for order confusions. Yet, logically, we see this is too restrictive: contour, pitch, and temporal order are interwoven in a pattern context. Furthermore, there is experimental evidence that contour and the pitch separations within an auditory pattern contribute to "streamability" and order confusions at faster rates (Jones et al., 1978). In short, the absolute-time-threshold concept may be less useful in explaining causes of order confusions in complex serial contexts than in the simple two-event case (see Jones, in press, for a review).

In serial patterns, people pick up relationships between unfolding events, and the degree to which they can do this determines their skill at detecting order reversals. To be sure, fast pattern rates will render a sequence of tonal transformations more difficult to attentionally track. But predictability in both contour and pitch-interval relationships will also determine the degree to which the listener can attentionally track, anticipate, and cope with unexpected tonal relationships (Jones, 1976).

In this respect, it is significant that all note-to-note time periods in Experiment 1 were comfortably longer (350msec SOA) than the threshold time values discussed 
Table 1

Mean Proportion Correct Recognition in Experiment 1 (Slow Rate) as a Function of Rules, Number of Contour Changes, and Pattern Shape

\begin{tabular}{|c|c|c|c|c|c|c|c|}
\hline \multirow{2}{*}{$\begin{array}{l}\text { Contour } \\
\text { Changes }\end{array}$} & \multicolumn{3}{|c|}{ Rule Invariant } & \multicolumn{3}{|c|}{ Rule Variant } & \multirow[b]{2}{*}{ Mean } \\
\hline & Symmetric & Asymmetric & Mean & Symmetric & Asymmetric & Mean & \\
\hline 0 & .89 & .93 & .91 & .93 & .91 & .92 & .91 \\
\hline 1 & .91 & .89 & .90 & .85 & .92 & .89 & .90 \\
\hline 2 & .86 & .89 & .87 & .86 & .84 & .85 & .86 \\
\hline 3 & .85 & .77 & .81 & .82 & .82 & .82 & .82 \\
\hline Mean & .88 & .87 & .87 & .87 & .87 & .87 & .87 \\
\hline
\end{tabular}

above. Experiment 2 was designed to further explore listeners' use of contour and pitch-interval relationships in patterns with faster rates. To accomplish this, the patterns of Experiment 1 were subjected to a time transposition that rendered them twice as fast $(\mathrm{SOA} \times 1 / 2)$. We anticipated that these faster patterns would produce more order confusions than those in Experiment 1. But of greater interest was the extent to which the variables of contour change, rule structure, and contour shape would produce similar effects in these faster sequences.

\section{Method}

Subjects. The subjects were 24 Ohio State University students, who volunteered to participate in a psychology experiment for course credit or for payment ( $\$ 2$ per hour). Each had had at least 2 years of musical experience within the past 5 years, and none had served in Experiment 1. Twelve subjects were assigned randomly to each of two counterbalance orders. (Three subjects did not perform above chance levels and were omitted from the analyses.)

Design and Procedure. The design and procedure were identical in every respect to those of Experiment 1.

Stimulus materials. Sixty-four 8-tone standard patterns that were melodically identical in every respect to those described for Experiment 1 were constructed. The single difference between sequences in Experiment 2 and those in Experiment 1 involved presentation rate: all SOAs in the patterns of Experiment 2 were $175 \mathrm{msec}$ based upon a 150 -msec tonal duration and a 25 -msec off time between successive tones.

Construction of comparison sequences for the $2 \mathrm{AFC}$ task and recording details remained the same as described for Experiment 1.

\section{Results and Discussion}

Figure $3 \mathrm{~b}$ shows the mean proportion of correct pattern recognition as a function of contour change and rule structure (collapsed over the contour symmetry variable and counterbalance order, both nonsignificant effects). Again, the most important finding was that contour complexity had large and detrimental effects on judgments $[\mathrm{F}(3,69)=5.98, \mathrm{MSe}=.026, \mathrm{p}<.005]$. As number of contour changes increased, PC again declined for both rule-invariant and rule-variant patterns. There was no significant interaction of rule with contour change $[F(3,69)$ $\cong 1.00]$.

However, at this faster rate, rule-invariant patterns elicited better performance than melodies having less predictable rule structure (rule variant) $[\mathrm{F}(1,23)=21.86$, $\mathrm{MSe}=.013, \mathrm{p}<.001]$. In this regard, these data differ from those reported for the slower sequences of Experiment 1 , where no overall effect of rule structure was evident. These findings suggest that both the amount of time and the presence of pitch and contour invariances are important for order retention.

Although rule invariance did help performance at these rates, the benefit of rule structure did not extend to higher order rule recursion. The fact that contour symmetry did not significantly interact with rule structure is particularly difficult for Restle's theory. Rule-recursive patterns (symmetrical, rule invariant) did not seem to cement serial order into simple codes. Table 2 presents mean PC as a function of contour symmetry and rule level for the various levels of contour change. As these data indicate, contour symmetry generally had remarkably little effect. In fact, there was a slight, but not significant, tendency for asymmetric patterns to produce better performance than did symmetric ones $[\mathrm{F}(1,23)=2.86, \mathrm{MSe}=.024$, $\mathrm{p} \cong .10]$.

The symmetry variable failed to interact with rule level and did not even produce a three-way interaction with rule level and contour change. Recall that such a three-way interaction did appear in Experiment 1, where it was interpreted as qualified support for a rule-recursion notion. In short, neither the global symmetry hypothesis nor the rule-recursion hypothesis received support from these data.

Differences in the results of Experiments 1 and 2 suggested a combined analysis of data from both experiments. A main effect for rate $[F(1,44)=5.51, \mathrm{MSe}=.17$, $\mathrm{p}<.025$ ] verified that the faster rate of Experiment 2 significantly lowered overall recognition performance. A rate $\times$ rule interaction $[\mathrm{F}(1,44)=12.65, \mathrm{MSe}=.01$, $\mathrm{p}<.001]$ also indicated that the faster rate predisposed listeners toward some kinds of structural information and not others. In particular, rule-invariant patterns were easier than rule-variant ones, showing that the presence or absence of rule predictability was more critical at faster rates than it was at slower rates. Other structural variables, including those of contour shape and number of changes, however, were unaffected by the rate variable. That is, there were no significant interactions of these structural variables with rate.

\section{GENERAL DISCUSSION}

The pattern of results from Experiments 1 and 2 is quite straightforward. It establishes three important new facts. These have to do with the impact of contour, rule invariance, and pattern shape on order retention. 
Table 2

Mean Proportion Correct Recognition in Experiment 2 (Fast Rate) as a Function of Rules, Number of Contour Changes, and Pattern Shape

\begin{tabular}{ccccccccc}
\hline \multirow{2}{*}{$\begin{array}{c}\text { Contour } \\
\text { Changes }\end{array}$} & \multicolumn{3}{c}{ Rule Invariant } & & \multicolumn{3}{c}{ Rule Variant } \\
\cline { 2 - 4 } \cline { 6 - 7 } & Symmetric & Asymmetric & Mean & & Symmetric & Asymmetric & Mean & Mean \\
\hline 0 & .86 & .87 & .86 & & .84 & .84 & .84 & .85 \\
1 & .84 & .86 & .85 & & .75 & .80 & .78 & .81 \\
2 & .76 & .86 & .81 & & .77 & .69 & .73 & .77 \\
3 & .76 & .82 & .79 & & .72 & .76 & .74 & .77 \\
Mean & .81 & .85 & .83 & & .77 & .77 & .77 & .80 \\
\hline
\end{tabular}

A pattern with multiple changes in pitch direction simply provides a listener with a more complex melodic object. Yet this in itself fails to explain why more changes should produce more order confusions. The answer is undoubtedly tied to the intimate connection between contour and temporal order. An unbroken pitch trajectory preserves ordinal information within that segment, and so a temporal reversal of constituent tones is likely to change the contour. The simplest explanation for effects of contour complexity, then, is that a change in contour is more noticeable in patterns with fewer contour changes than in ones with many changes. Clearly, for this explanation to hold, we must assume that people are sensitive to contour information and, in fact, partially rely upon it to make judgments about the temporal order of tones. The present data, as well as those reported by Boltz and Jones (1985), support this view. Boltz and Jones found that melodic reproductions become less accurate as the number of contour changes increases. They suggest that contour change points function as salient melodic accents. If true, then, as more changes occur, so does the probability of accent anisochrony, a feature that would both contribute to disruption of the listener's dynamic attending and interfere with his/her recognition of an order reversal.

The fact that contour has such a marked effect upon order recognition, even at slow rates, is rather troublesome for Restle's (1970) theory. His approach ignores contour while emphasizing the influence of, for example, musical interval differences and rule recursion upon code complexity. Coding theories such as Restle's predict that melodies exhibiting rule invariance in tonal transformations should produce better order recognition than those that do not, and to some extent this was found to be true. A potential problem, however, is the fact that no differences between rule-variant and rule-invariant patterns were found at slower rates, where performance was generally quite good. Only at faster rates was the prediction clearly upheld. To accommodate this interaction, one could modify the coding approach by assuming that requisite coding time per tonal transformation is greater in rule-variant melodies than in invariant ones. However, there is another possible explanation for the rate $\times$ rule structure interaction that comes from the dynamic attending approach. According to the latter view, listeners do not attempt to encode all "registered" items. Instead, it is hypothesized that people respond to the unfolding of certain transformations over time and use this dynamic information to guide attending, in an anticipatory way, toward important future points in the pattern (e.g., contour accents) (cf. Jones, 1981a). The difference between the dynamic attending approach and coding models centers on the former's assumption that people use the pattern's time structure, namely, its tempo and rhythm, to guide attending over time. In contrast, coding models tend to assume static rule codes (of varying complexity) (see Jones, in press, for a discussion). Under the assumption that listeners do dynamically anticipate future tonal relationships, the attending model suggests that additional time to improve performance is required only when anticipations prove incorrect. This is more likely to occur with rule-variant patterns, and therefore faster rates will be more detrimental for these sequences. The difference is a subtle, but important, one, and the present data do not distinguish between the two explanations.

The third major finding of the present experiments is that contour shape did not significantly affect serial-order retention. Symmetrical patterns, whether born of a rulerecursion formula or not, were generally no easier than asymmetrical ones. This finding suggests that theories arguing for the inherent simplicity of hierarchical (binary) rule trees must be qualified. Instead, the results are clear in suggesting that the number of contour changes is more critical to order confusions than contour shape-an issue that merits further experimental evaluation.

In summary, the determinants of temporal-order retention in complex auditory patterns are closely bound with the structure of the pattern itself. Time is part of this structure in that a pattern is defined by both its tempo and rhythm. But contour change and melodic rule variability also contribute importantly to the temporal coherence of tonal sequences.

\section{REFERENCES}

Boltz, M., \& Jones, M. R. (1985). Does rule recursion make melodies easier to reproduce? If not, what does? Manuscript submitted for publication.

Cuddy, L. L., Cohen, A. J., Mewhort, D. J. K. (1981). Perception of structure of short melodic sequences. Journal of Experimental Psychology: Human Perception and Performance, 7, 869-882.

Deutsch, D. (1972). Octave generalization and tune recognition. Perception \& Psychophysics, 11, 411-412.

DiVENYI, P. L., \& HIRSH, I. J. (1974). Identification of temporal order in three-tone sequences. Journal of the Acoustical Society of America, 56, 144-151. 
DOWLING, W. J. (1972). Recognition of melodic transformations: Inversion, retrograde and retrograde inversion. Perception \& Psychophysics, 12, 417-421.

DowLING, W. J. (1978a). Listeners' successful search for melodies scrambled into several octaves. Journal of the Acoustical Society of America, 64, S146.

Dowling, W. J. (1978b). Scale and contour: Two components of a theory of memory for melodies. Psychological Review, 85, 341-354

DowLING, W. J., \& BARTLETT, J. C. (1981). The importance of interval information in long-term memory for melodies. Psychomusicology, 1, 30-49

Dowlung, W. J., \& Fujitani, D. S. (1971). Contour, interval, and pitch recognition in memory for melodies. Journal of the Acoustical Society of America, 49, 524-531.

FAY, W. H. (1966). Temporal sequence in the perception of speech. The Hague: Mouton.

Greeno, J., Simon, H. A. (1974). Processes for sequence production. Psychological Review, 81, 187-198.

HiRsh, I. J. (1959). Auditory perception of temporal order. Jourmal of the Acoustical Society of America, 31, 759-767.

HiRSH, I. J. (1974). Temporal order and auditory perception. In H. R. Moskowitz, B. Scharf, \& J. D. Stevens (Eds.), Sensation and measurement. Dordrecht, Holland: Reidel.

Hirsh, I. J., \& Sherrick, C. E. (1961). Perceived order in different sense modalities. Journal of Experimental Psychology, 62, 423-432.

IDSON, W. L., \& Massaro, D. W. (1978). A bidimensional model of pitch in the recognition of melodies. Perception \& Psychophysics, 24, 551-565.

JONES, M. R. (1974). Cognitive representations of serial patterns. In B. Kantowitz (Ed.), Human information processing: Tutorials in performance cognition. Potomac, MD: Erlbaum.

JoNEs, M. R. (1976). Time, our lost dimension: Toward a new theory of perception, attention, and memory. Psychological Review, 83, 323-355.

JoNeS, M. R. (1981a). Music as a stimulus for psychological motion: Part I. Some determinants of expectancies. Psychomusicology, 1 , 34-51.

JONES, M. R. (1981b). A tutorial on some issues and methods in serial pattern research. Perception \& Psychophysics, 30, 492-504.

JONES, M. R. (in press). Review paper on structural organization in events in time. In $\mathbf{J}$. Michon \& $\mathbf{J}$. Jackson-Roy (Eds.), Time, mind, and behavior. Heidelberg: Springer.

JONES, M. R., MASER, D. J., \& KIDD, G. R. (1978). Rate and structure in memory for auditory patterns. Memory \& Cognition, 6, 246-258.

Kallman, H. J., \& Massaro, D. W. (1979). Tone chroma is functional in melody recognition. Perception \& Psychophysics, 26, 32-36.

Massaro, D. W. (1972). Preperceptual images, processing time, and perceptual units in auditory perception. Psychological Review, 79. 124-145.
Massaro, D. W., Kallman, H. J., \& Kelly, J. L. (1980). The role of tone height, melodic contour, and tone chroma in melody recognition. Journal of Experimental Psychology: Human Learning and Memory, 6, 77-90.

REsTLE, F. (1970). Theory of serial pattern learning: Structural trees. Psychological Review, 77, 481-495.

Restle, F., \& BRown, E. R. (1970). Organization of serial pattem learning. In G. H. Bower (Ed.), The psychology of learning and motivation: Advances in research and theory (Vol. 4). New York: Academic Press.

Stroud, J. M. (1955). The fine structure of psychological time. In $\mathbf{H}$ Quastler (Ed.), Information theory in psychology. Glencoe, IL: Free Press.

Thomas, I. B., Hill, P. B., Carroll, F. S., \& Garcia, G. (1970). Temporal order in the perception of vowels. Journal of the Acoustical Society of America, 48, 1010-1013.

VAN NOORDEN, L. P. A. S. (1975). Temporal coherence in the perception of tone sequences. Doctoral thesis, Eindhoven University of Technology, Eindhoven, The Netherlands.

WARREN, R. M. (1974). Auditory temporal discrimination by trained listeners. Cognitive Psychology, 6, 237-256.

Warren, R. M. (1976). Auditory illusions and perceptual processes. In N. J. Lass (Ed.), Contemporary issues in experimental phonetics. New York: Academic Press.

\section{NOTES}

1. In rule-invariant patterns, the compound $\mathrm{CN}^{\mathrm{j}}$ operation involves application of $\mathrm{C}$ and then $\mathrm{N}^{\mathrm{j}}$ to the same argument. Since the $C \mathrm{~N}^{\mathrm{j}}$ operation necessarily produces a contour reversal, any $\mathrm{N}^{\mathrm{j}}$ rule following a $\mathrm{CN}^{\mathrm{j}}$ operation in a sequence will change in sign. Thus, for example, the rule-recursive pattern of $\mathrm{N}^{+3} \mathrm{CN}^{-2} \mathrm{~N}^{-3}$ in Figure 2 contains a final $\mathrm{N}^{-3}$ rule that is reversed in sign from the initial $\mathrm{N}^{+3}$ rule.

2. A musical scale consists of a set of frequencies (subjectively, pitches) spanning 1 octave. An octave reflects frequency doubling; that is, octaves are logarithmically equal frequency intervals. A diatonic major scale is constituted of a serial arrangement of two other kinds of intervals between tones: the semitone, $s$, and the whole tone, $w$. A semitone is the smallest interval in traditional Western music, and 1 whole tone equals 2 semitones. For example, the $\mathrm{C}$ major scale in the fourth octave $\left(\mathrm{C}_{4}=\right.$ middle $\mathrm{C}$ on the piano) consists of the following notes:

$$
\mathrm{C}_{4} \text { (w) } \mathrm{D}_{4} \text { (w) } \mathrm{E}_{4} \text { (s) } \mathrm{F}_{4} \text { (w) } \mathrm{G}_{4} \text { (w) } \mathrm{A}_{4} \text { (w) } \mathrm{B}_{4} \text { (s); }
$$

$C_{5}$ is 1 semitone higher than $B_{4}$, and its frequency $(524 \mathrm{~Hz})$ is double the frequency of $\mathrm{C}_{4}(262 \mathrm{~Hz})$.

(Manuscript received September 3, 1984; revision accepted for publication February 19, 1985.) 